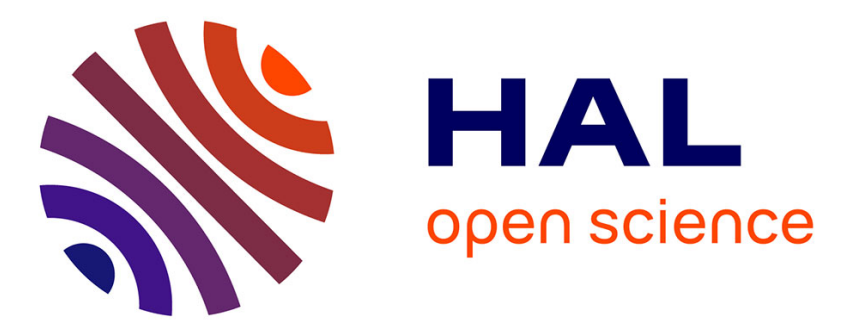

\title{
Passenger-Centric Metrics for Air Transportation Leveraging Mobile Phone and Twitter Data
}

\author{
Aude Marzuoli, Philippe Monmousseau, Eric Féron
}

\section{To cite this version:}

Aude Marzuoli, Philippe Monmousseau, Eric Féron. Passenger-Centric Metrics for Air Transportation Leveraging Mobile Phone and Twitter Data. ICDMW 2018, IEEE International Conference on Data Mining Workshops, Nov 2018, Singapour, Singapore. 10.1109/ICDMW.2018.00091 . hal-02078088

\section{HAL Id: hal-02078088 \\ https://hal-enac.archives-ouvertes.fr/hal-02078088}

Submitted on 28 Mar 2019

HAL is a multi-disciplinary open access archive for the deposit and dissemination of scientific research documents, whether they are published or not. The documents may come from teaching and research institutions in France or abroad, or from public or private research centers.
L'archive ouverte pluridisciplinaire HAL, est destinée au dépôt et à la diffusion de documents scientifiques de niveau recherche, publiés ou non, émanant des établissements d'enseignement et de recherche français ou étrangers, des laboratoires publics ou privés. 


\title{
Passenger-centric metrics for Air Transportation leveraging mobile phone and Twitter data
}

\author{
Aude Marzuoli, Philippe Monmousseau, Eric Feron \\ School of Aerospace Engineering, Georgia Institute of Technology, Atlanta, Georgia 30332-0250 \\ École Nationale de l'Aviation Civile, Toulouse, France \\ Email: amarzuoli3,pmonmousseau3, feron@gatech.edu
}

\begin{abstract}
This paper aims at presenting a detailed analysis of domestic air passengers behavior during a major airtraffic disturbance, from two complementary passenger-centric perspective: a passenger mobility perspective and a passenger social media perspective. By leveraging over 5 billion records of mobile phone location data per day from a major carrier in the United States, passenger mobility can be reliably analyzed, no matter which airline the passengers fly on or which airport they fly to and from. Such information is currently unavailable to the major aviation stakeholders at such scale and can be used to establish performance benchmarks from a passenger's perspective. Combining it with a Twitter analysis provides a more detailed and passenger-focused analysis than the traditional flight-centric measurements used to evaluate the overall system performance. More generally, these two passenger-centric analysis could be implemented in real-time for a daily evaluation of the Air Transportation System, enabling a faster analysis of the impact of major disruptions, whether due to meteorological conditions or system failures.
\end{abstract}

\section{INTRODUCTION}

The Air Transportation System is a complex interconnected system that carried more than 631 million passengers on domestic flights in the United States in 2010 according to the Bureau of Transportation Statistics (BTS) [1]. Passengers are at the core of this system and, yet, limited quantitative information about passenger movements is publicly shared. Each aviation stakeholder only has access to a partial view of the passenger-side of things. Passenger information is airline proprietary information. Each airline therefore has a partial view of passenger movements on board aircraft and on the ground (from check-in kiosks and counters to boarding the aircraft). Airports gather customs or security records, shuttle traffic, parking occupancy, sometimes measure queue lengths, while third-parties collect online traces through WiFi hotspots and Bluetooth beacons [2]. Therefore, a system-wide datadriven picture of passenger behavior remains unavailable. The BTS provides aggregated passenger data per market but no granular information. Passenger surveys conducted by airports or airlines, while very detailed, remain limited to small samples of passengers and short time periods, and may not be representative.

Airports constitute the main bottlenecks of the air transportation system. The management of different airport processes is shared between various stakeholders, from airlines to government, airport authorities and third parties, who do not necessarily rely on each other to make decisions that may affect others [3]. Passengers' satisfaction is largely driven by their experience at the airport, and this experience is the result of the combined control exerted by many stakeholders.

For several years, NextGen [4] in the United States and SESAR [5] in Europe have been advocating a shift from flightcentric metrics to passenger-centric metrics for the performance evaluation of the Air Transportation System [6] [7]. Multiple studies have shown the disproportionate impact of airside disruptions on passenger door-to-door journeys. Flight delays do not accurately reflect the delays imposed upon passengers' full multimodal itinerary. Cook and al. [8] designed propagation-centric and passenger-centric performance metrics, and compare them with existing flight-centric metrics. Bratu et al. [9], calculated passenger delay using monthly data from a major airline operating a hub-and-spoke network. They show that disrupted passengers, whose journey was interrupted by a capacity reduction, are only $3 \%$ of the total passengers, but suffer $39 \%$ of the total passenger delay. Wang [10] showed that high passenger trip delays are disproportionately generated by canceled flights and missed connections. 9 of the busiest 35 airports cause $50 \%$ of total passenger trip delays. Congestion, flight delay, load factor, flight cancellation time and airline cooperation policy are the most significant factors affecting total passenger trip delay. Both NextGen and SESAR aim at improving the predictability and resilience of the Air Transportation System, which includes reducing door-to-door travel time for passengers. Currently, simply measuring doorto-door travel time remains a major hurdle.

However, most individuals now carry a cell phone, and heavily use it through out the day. Phone carriers collect Call Detail Records (CDR), indicating when an individual makes a phone call, texts, or browses online, as well as the individual's approximate location when doing so. Please note that such records belong to the carriers and are generally not publicly available. Only in a few instances have partial data sets been anonymized and released for research applications. As early as 2008, Bayen et al. demonstrated the use of smartphones to monitoring highway traffic in the Bay Area [11]. Gonzalez et al. showed how large scale studies of CDRs can help understand individual mobility patterns [12]. Blondel et al. provided a thorough survey [13] of applications of mobile phone data from mobility, to urban planning and help towards development in Africa for instance [14] [15]. De Montjoye et al. built a Python toolbox to help researchers analyze, 
visualize and build robust features from mobile phone data [16]. Douglass et al. provided high resolution population estimates from mobile phone data [17]. CDRs can be used to identify home and work locations reliably and allow the extraction of additional frequent locations, activity travel diary validated comparing them to household surveys [18]. Toole et al. focused on using CDRs for urban planning, and in particular travel-demand estimation to provide validated origindestination matrices on the ground and road usage patterns [19].

Another popular source of data previously used for studying large-scale behaviors is social media, in particular Twitter. With more than 68 millions active users in the United Stated[20], Twitter is an important pool of user-created data that is still not fully leveraged. Twitter has already been the main focus of many studies, including studies on its network topology by Java et al. [21], Krishnamurthy et al. [22] and Huberman et al. [23], as well as more recent studies by Palen et al. on how Twitter is being used during natural disasters [24], [25] [26].

The main contribution of the paper is to provide tools for the analysis of the impact of disruptions on the air traffic system from two different passenger-centric perspectives: a passenger mobility perspective using mobile location data from a major US phone carrier and a passenger social media perspective based on Twitter data. These tools are implemented and tested a posteriori in the case of the bomb cyclone that hit the Northeast part of the United States in January 2018, causing the closure of Kennedy International Airport (JFK) and severe capacity decreases at Logan International Airport (BOS), Newark Liberty International Airport (EWR) and LaGuardia Airport (LGA). The passenger mobility perspective is based on a previous experimental proof [27] that mobile phone data enables the identification of domestic air passengers and supports the analysis of their behavior, under both nominal and degraded conditions. Combined with a passenger social media behavior perspective, these two analysis yield a better understanding of the impact of this bomb cyclone than the traditional flight-centric data coming from the BTS database.

The paper is organized as follows. Section II describes the bomb cyclone and its impact on flight operations, leveraging publicly available on-time performance data from the Bureau of Transportation Statistics. Section III offers a passengercentric perspective in this paper, focused on passenger mobility, supported by mobile phone cell-tower location data from a major US carrier. Section IV provides a second passengercentric perspective, focused on passenger travel experience, using publicly available Twitter data. Section V draws the conclusions of the study and provides future research perspectives.

\section{The Bomb Cyclone AND ITS IMPACT ON AiR OPERATIONS}

From January 2nd to January 6th 2018, a massive blizzard nicknamed "Bomb Cyclone" disrupted the Eastern Coast of the United States with a peak on January 4th. More than
90 percent of LGA flights, more than 70 percent of Newark Liberty flights and 20 percent of JFK flights were announced to be cancelled on January 4th. Both JFK and LGA airports were closed for safety measures due to the weather conditions [28] [29]. Port Authority closed JFK airport at 10:45 am on January 4th, expecting reopening at $3 \mathrm{pm}$. At $2 \mathrm{pm}$, the reopening was pushed to $8 \mathrm{pm}$. At $6 \mathrm{pm}$, it was pushed a second time to the next day, January 5th, at 7 am. On January 7 th, the record low temperatures led to water pipes breaking at JFK Terminal 4, forcing a partial evacuation and flooding hundreds of luggage.

\section{A. Overall impact on the United States}

The on-time performance measures of the BTS provide flight-level information for each day, indicating for all scheduled flights, whether a flight was canceled or delayed, and comparing scheduled versus actual departure and arrival times. In this section, we selected the top 45 airports in terms of traffic volume in the continental United States and extracted all traffic between these airports. Given the hub-and-spoke structure of the airport network, this represents the majority of domestic operations.

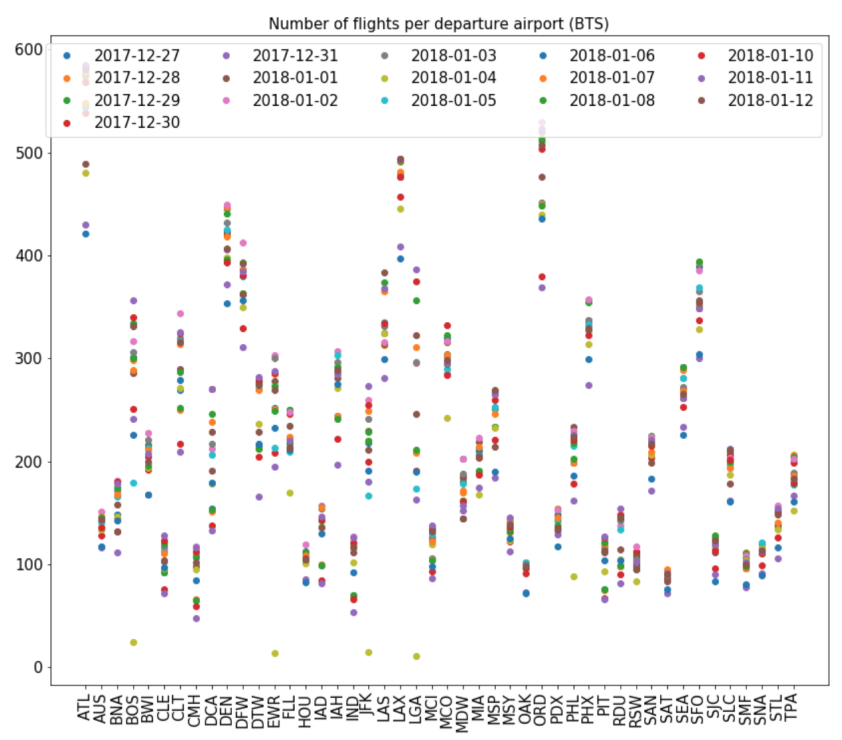

Fig. 1: Number of flights per departure airports (BTS)

The number of flown flights, aggregated by departing airports each day between December 27th, 2017 and January 12th, 2018, is shown in Figure 1. This initial flight-centric perspective confirms the major impact the bomb cyclone had on four airports in particular: BOS, EWR, LGA and JFK. The volume of flights on January 4th is an extreme outlier for these airports, which are amongst the busiest in the United States, and is still lower than usual on January 5th.

\section{B. Focus on the North East}

Figure 1 highlighted the impact of the bomb cyclone on four major airports of the North East of the United States, namely JFK, LGA, EWR and BOS. Figure 1 highlights the abnormal flight operations on January 4th (each of these airports had less 
than 30 flights overall) and emphasizes the two-day recovery period needed to return to a normal volume of operations.

However, the recovery in terms of schedule adherence and delays took longer, as depicted in Figure 2, showing the number of delayed flights at these airports. First, there are almost no delayed flights on January 4th since the vast majority was cancelled. The recovery period took about five days. Figure 3 presents the average flight delay per day at each airport at departure and arrival. The average departure delay shows different recovery profiles. LGA airport had its peak departure delay (across only 11 flights) on January 4th, while it was on January 5th for the other airports. Moreover, on the worst day of the Bomb cyclone, January 4th, at BOS, the average flight delay spiked to over 11 hours, for the only 3 flights that landed. For the other three airports, the peak arrival delay is on January 5 th.
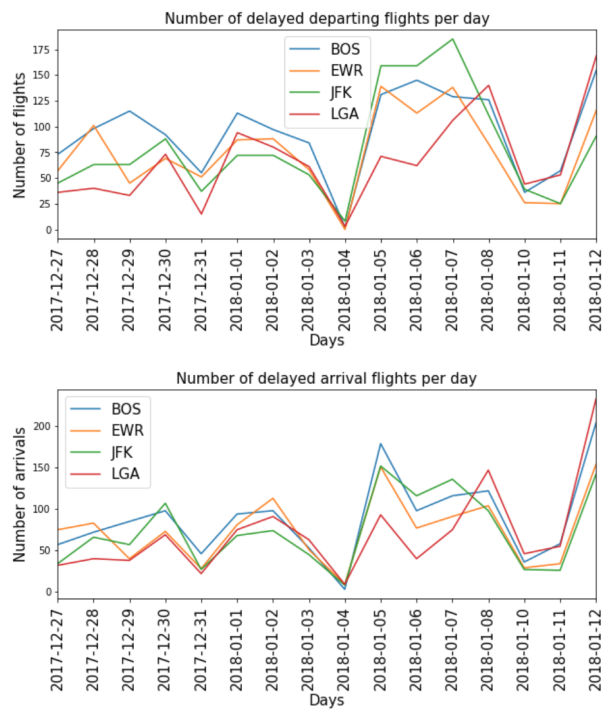

Fig. 2: Number of delayed flights per day.

From the BTS data, we can evaluate the quantitative impact of the bomb cyclone on flight traffic. Yet, we cannot fully apprehend the disproportionate impact of the bomb cyclone on passenger experience.

\section{BOMB CYCLONE FROM MOBILE LOCATION DATA}

In this section, the method of passenger selection validated in [27] is implemented and analyzed for the time period covering the bomb cyclone.

\section{A. Global view of domestic passengers experience at airports}

The top 45 airports in terms of traffic were chosen for this study and latitude/longitude bounding boxes were created for each of them. On a daily basis, 5 billion records are collected by the carrier each time a phone connects to the cellular network and an approximate location is obtained from cell tower triangulation. A record consists in an anonymized user id, a time stamp and the approximate latitude and longitude of the user. Passengers are identified if they have a cell phone
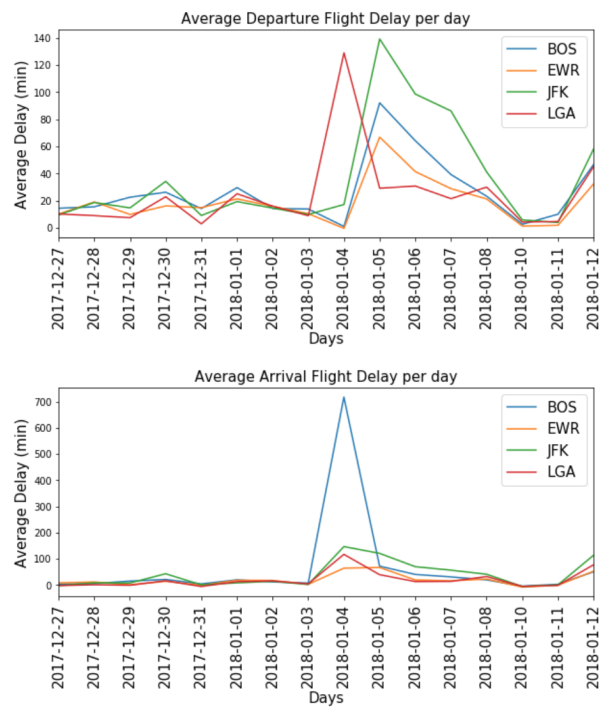

Fig. 3: Average flight delay per day.

record located within the bounding boxes of at least two different airports, provided these airports are not in the same metropolitan area. Once the passengers are detected, only the initial and final time stamps within each bounding box are kept in order to have a reliable estimate of the time spent by the passengers in each airport.

The number of passengers per day for these airports using this method is represented in Figure 4. From this plot, the same four northeastern airports are noticeable as outliers on January 4th and 5th, 2018. This simple observation indicates that, from a passenger perspective, the peak of the bomb cyclone's impact was not solely located on January 4th as the BTS data shows.

Making a box plot visualization of the time spent at airports yields a more condensed way of comparing the performance of the airports in terms of passenger time spent within the airport. Figure 5, which shows the average and quartile distribution of the time spent by passengers at each airport on January 2nd, 2018, at departure or arrival. As expected, passengers typically spend more time at departure than at arrival. January 2nd is selected as a fairly uneventful day, to portray the usual performance of each airport from a passenger's perspective. For example, LAX and MCO have the highest average time spent by passengers at departure, with 130 minutes, but LAS has the highest standard deviation, with 71 minutes. At arrival, the worst performer is DFW with 87 minutes on average.

Compare Figure 5 for January 2nd with Figures $6 \& 7$ showing Jan 4th and 5th respectively. On January 4th, the number of passengers at BOS, EWR, JFK and LGA is very small. Between January 2nd and 4th, passengers spent in average less time at departure at the impacted airports (about 10 percent) but with a wider distribution. Visually, this can be illustrated as the box plot sinking and widening compared to its normal state. While the average time spent at departure is similar to that of January 2nd, the standard deviation is about 20 percent higher for these four airports. On January 5th, 


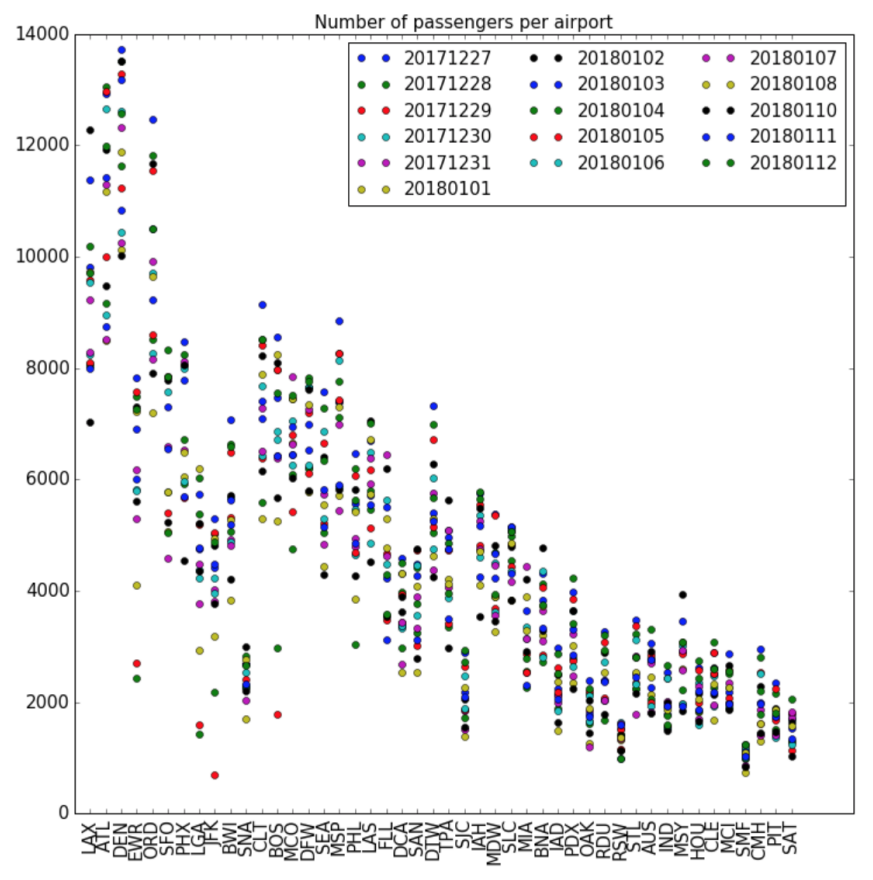

Fig. 4: Number of passengers per airport.
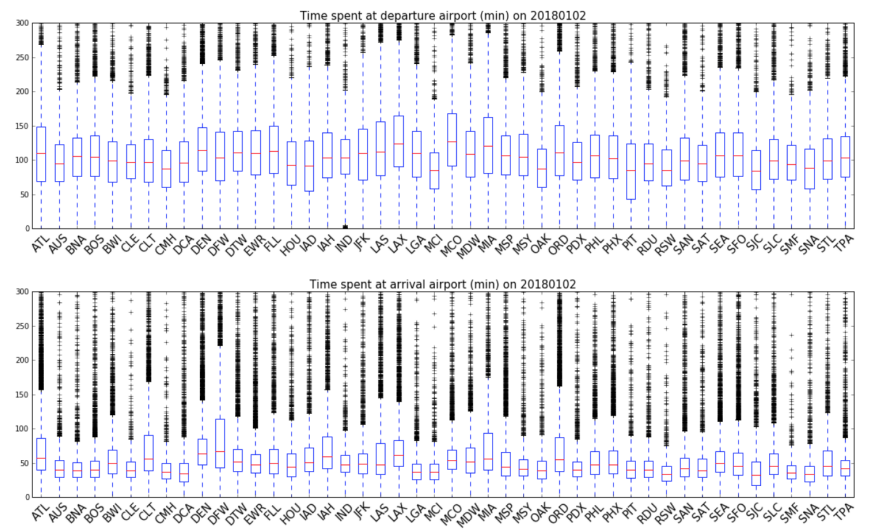

Fig. 5: Time spent at airports by passengers on January 2nd.

when there were less cancellations but a peak in flight delay, we observe a peak in time spent at departure. For instance, at JFK, on January 2nd, a departing passenger spends 109 minutes on average, with a standard deviation of 59 minutes. This is a good performance compared to the other airports in the United States. But on January 5th at JFK, the average time at departure for passengers jumps to 194 minutes and the standard deviation to 98 minutes.

These new plots and new methods have confirmed that mobile phone data do pinpoint airports that are going through major disturbances by establishing a reliable benchmark of their performance from the passengers perspective.

\section{B. Analysis at each airport in the North East}

Once the most impacted airports are identified from comparing with the top airports, a more specific analysis can be
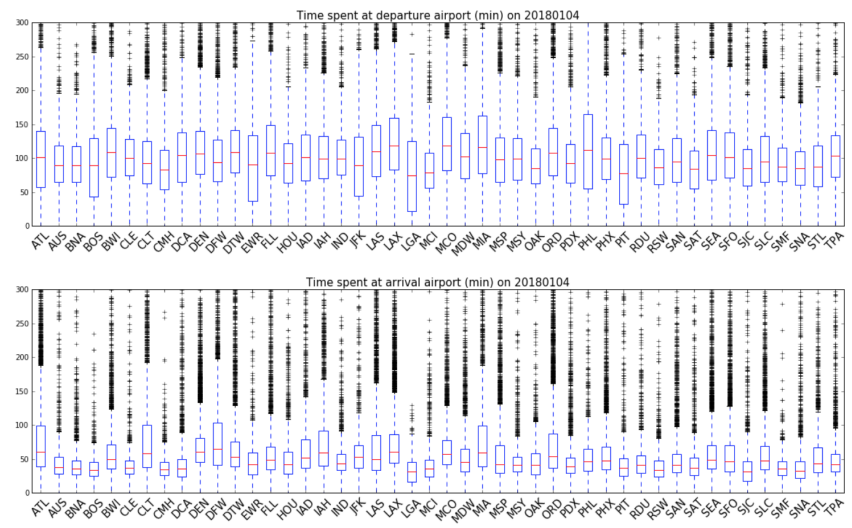

Fig. 6: Time spent at airports by passengers on January 4th.
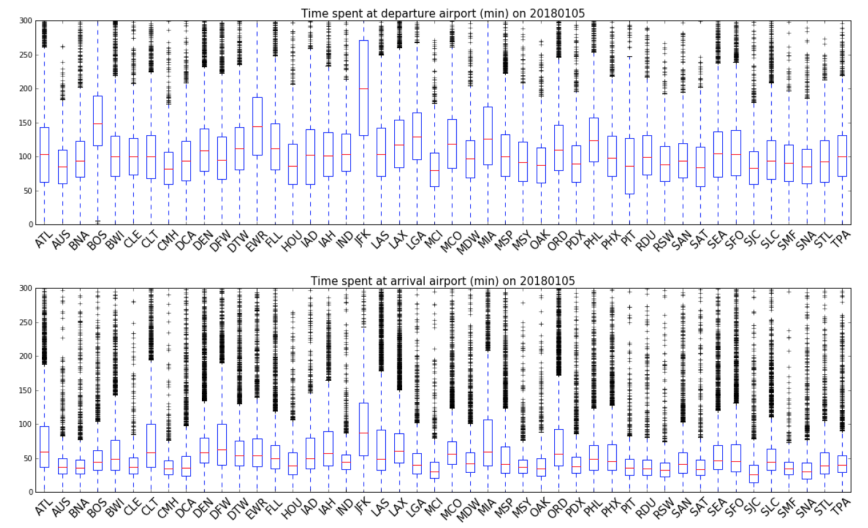

Fig. 7: Time spent at airports by passengers on January 5th.

conducted to better evaluate if this disturbance impacted each of these airports differently.

1) Visitors: First, we examine the behavior of users visiting the airport, i.e. people who were within the bounding box of an airport. These visitors includes passengers, airport staff, taxi drivers, and anyone driving by as well. This approach is useful to know if the disturbance only affected passengers or a wider group. Figure 8 shows the evolution of the number of visitors per day over two weeks around the bomb cyclone for the four impacted airports. Each airport considered typically employs between 15,000 and 40,000 people, as airport staff. Thousands of domestic and international passengers transit through each airport. Friends and family drop off and pick up passengers. Several airports are located along major roads or highways, and because location data is noisy, pings might be recorded within bounding boxes around the airport. What matters here is not the absolute number but the relative changes day to day. The signal for passengers is much cleaner thanks to a more elaborate filtering.

The drop on January 4th is clearly visible for all four impacted airports, with different recovery profiles. EWR had the fastest recovery while JFK's recovery was slower and started a day later.

Plotting the distribution of the time spent at these airports by 


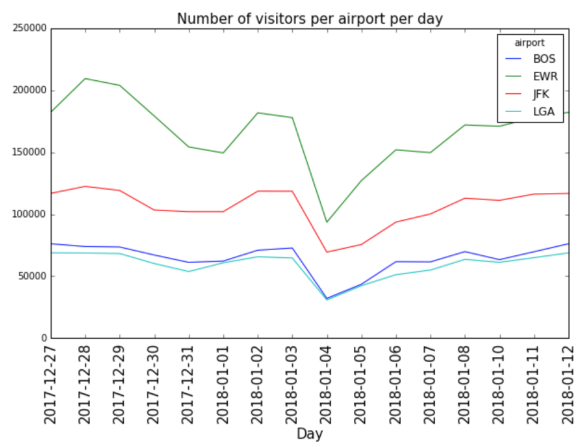

Fig. 8: Evolution of the number of visitors for Eastern airports.

visitors every day yields some striking patterns. From Figure 9 , the average time spent by visitors is fairly consistent over the days except on January 4th and 5th. These plots show that even the number of visitors dropped during the bomb cyclone, most likely because the snow levels made access to these airports difficult. The peak is most visible for BOS.

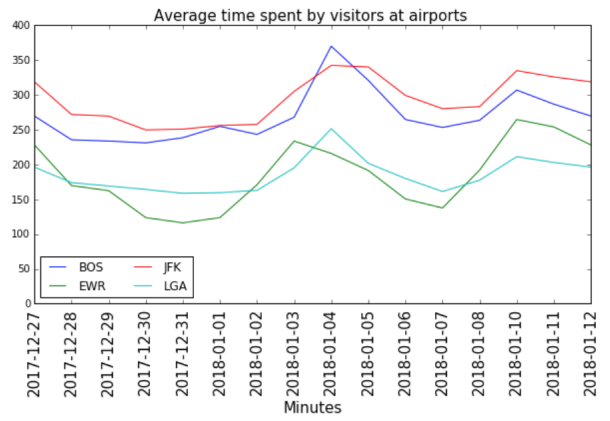

Fig. 9: Average time spent by visitors at airports most impacted by the Bomb Cyclone.

This visitor-centric view give us a better insight on the impact of any disturbance for different airports. During the the bomb cyclone, both passengers, who flew in or out of the airport, and visitors were impacted. The difference of impact between the different airports for visitors also illustrates how the airports' access routes had an effect or were impacted by the anomaly.

2) Passengers: The box plots used to create a performance benchmark from a passenger's perspective proposed in section III-A are useful to pinpoint impacted airports, and they can be used in a different configuration to gain additional insight on the differences between normal and disrupted behavior at each airport. Figures 10 and 11 shows the evolution across days of the average and standard deviation of the time spent at departure and arrival for passengers at the airports most impacted by the Bomb cyclone. The differences noted previously become obviously visible in terms of averages: on January 4th, at JFK, EWR, LGA and BOS, there is a small decrease in the average time spent at departure with a wider distribution for smaller waiting times followed by an important increase of the average time spent on January 5th with an increased distribution spread

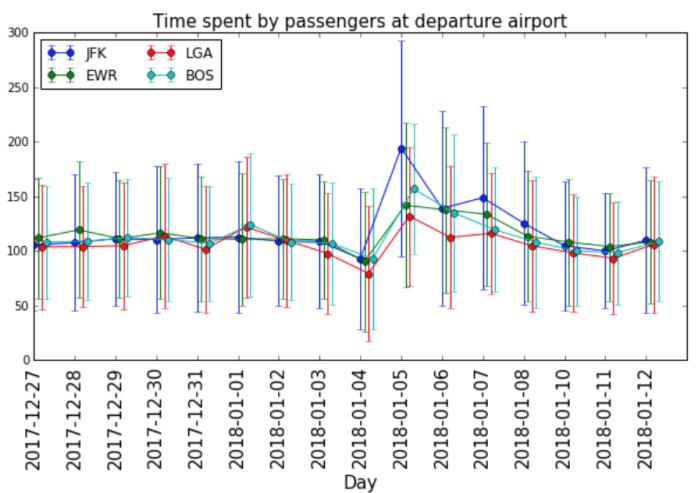

Fig. 10: Average and standard deviation of time spent by passengers at departure airports.

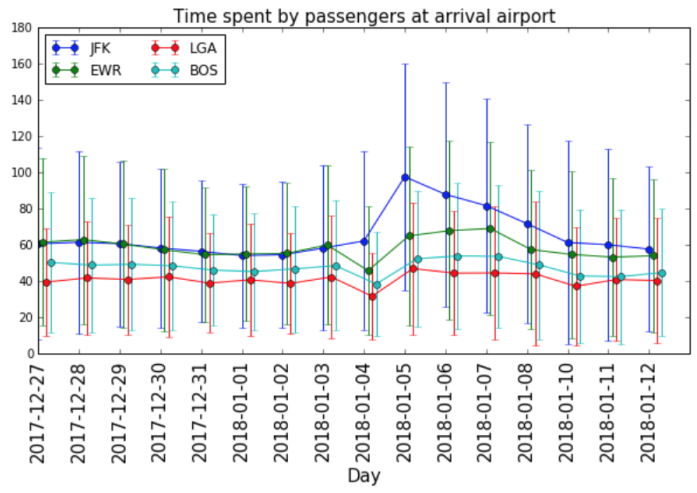

Fig. 11: Average and standard deviation of time spent by passengers at arrival airports.

as well. Regarding the time spent at arrival, the patterns are similar, although less marked. In terms of standard deviations, JFK has a large increase in the width of the time distribution starting January 5th for the time spent at departure and a four day recovery period for this parameter while LGA does not have this increase in width as well as a one day recovery period. EWR and BOS experience a lower increase in spread than JFK and they both have a three day recovery period. Overall, the recovery took longer at JFK than at EWR, LGA and BOS.

\section{BOMB CyCLONE ON TWITTER}

\section{A. Volume of tweets related to airlines/airports}

Using Twitter's developer APIn[30], we collected tweets related to airlines or airports handles (see Table I) over the same time period as in the previous sections. We created a database of tweets labeled by airline and by airport. Each entry of this database consists of the tweet ID, the time stamp , the text and the account handle used for the search.

First, we aggregate the volume of tweets per day and per handle and examine its evolution over time in order to understand the social impact of the bomb cyclone. Figure 12a illustrates that customers had a different experience depending 


\begin{tabular}{|c|c|}
\hline & Twitter handles \\
\hline Airlines & $\begin{array}{r}\text { @united, @Delta, @AmericanAir, @SouthwestAir, } \\
\text { @ SpiritAirlines, @VirginAmerica, @ JetBlue }\end{array}$ \\
\hline Airports & @JFKairport, @EWRairport, @BostonLogan, @LGAairport \\
\hline
\end{tabular}

TABLE I: Twitter handles used for gathering tweets

on which airlines they were flying. The most impacted airlines were Jetblue (B6) and Delta (DL). Figure 12b highlights how much worse the impact was at JFK airport compared to other North East airports, with a disproportionate amount of tweets from January 4th to January 14th. The peak of tweets is observed on January 7 th, when a terminal was flooded by a broken water pipe.

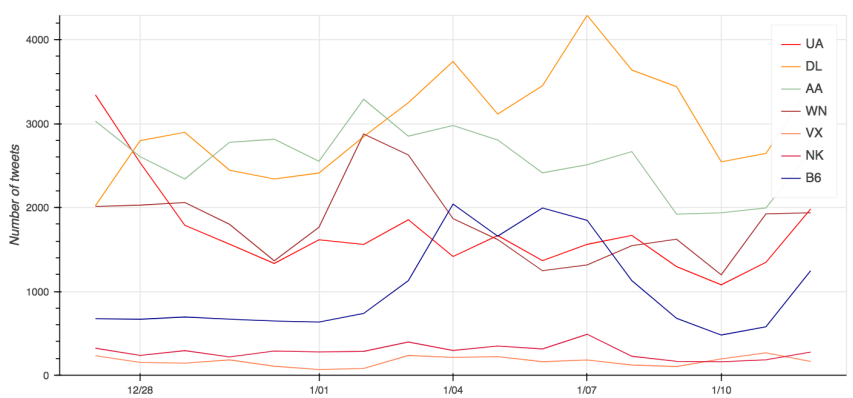

(a) Volume per airlines

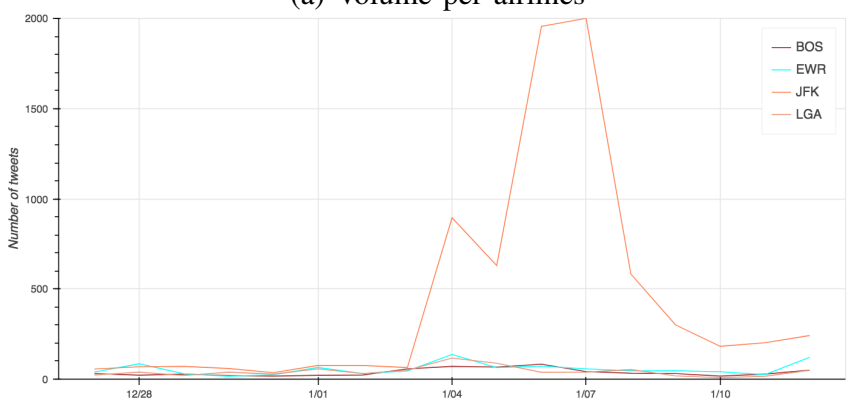

(b) Volume per airports

Fig. 12: Volume of tweets referring to airlines/airports aggregated by day

To obtain a more detailed picture of the situation on social media, we examine the tweets on an hourly basis. From Figure 13, we notice that Delta typically has a higher tweet volume over all days than other airlines, with a peak on January 7 th. JetBlue shows a large increase in tweets on January 4th in the afternoon, before steadying at a lower level, albeit higher than on normal days, for the following two days. The visualization of tweet volume is more striking in terms of airports aggregated per hour, see Figure 13b. From the normal small chatter common to the other airports, JFK becomes a huge source of tweets as soon as 6am on January 4th. And this source takes five full days before slowly disappearing, which is consistent with the phone location analysis presented previously in Section III.

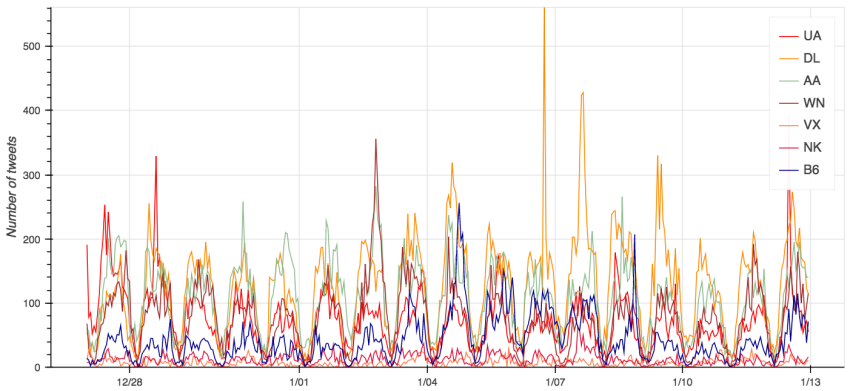

(a) Volume per airlines

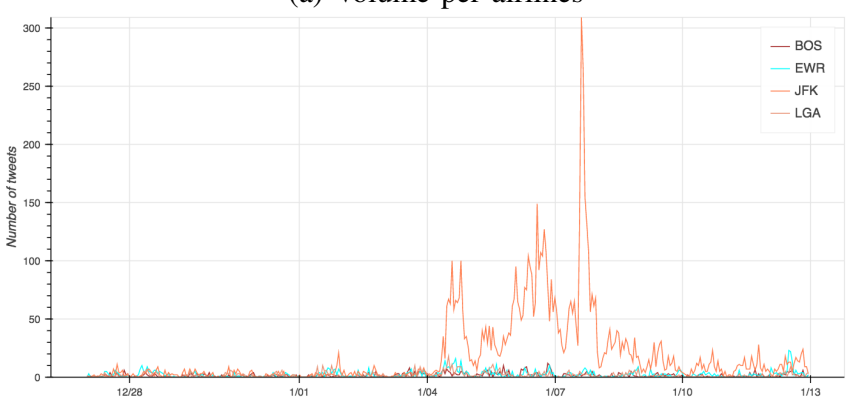

(b) Volume per airports

Fig. 13: Volume of tweets referring to airlines/airports aggregated by hour

\section{B. Tweets about delays and cancellations}

While monitoring tweet volume provides clues regarding the presence of anomalies, Twitter is most useful to obtain contextual information. Using simple filters based on the presence of keywords, one can get a better understanding of airlines' performance and overall passenger satisfaction. Filters on cancellation or delay related keywords yield some interesting results. The keywords used for these filters can be found in Table II.

\begin{tabular}{|c|c|}
\hline Filter & Keywords \\
\hline Cancellation & cancellation, cancel, cancelled, postponed \\
\hline Delay & delay, delayed, wait, waiting, late, postponed, hours \\
\hline
\end{tabular}

TABLE II: Keywords used for filtering tweets

Applying these filters and aggregating all airlines-related tweets reveals that cancellations had a greater impact than delays on passengers' social behavior as shown on Figure 14. The volume of cancellation-related tweets increases almost five-fold on Jan. 3rd, the day many cancellations were first announced given the weather forecasts, and keeps increasing on January 4th when the cancellations actually take place. The five day impact seen on the previous analysis is still visible on Figure 14a. Regarding the volume of delay-related tweets, the increase is less visible since the amount of delay actually decreased due to the increase of cancellations. However, the return to normal activity around January 10th is still clearly visible on Figure 14b. 


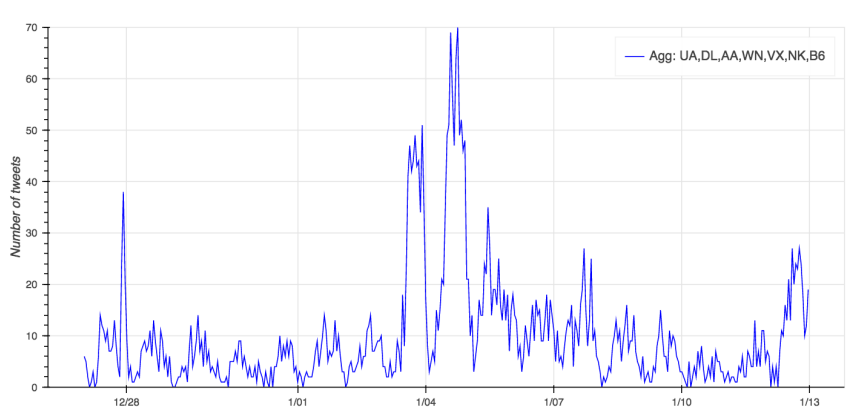

(a) Filtered with cancellation keywords

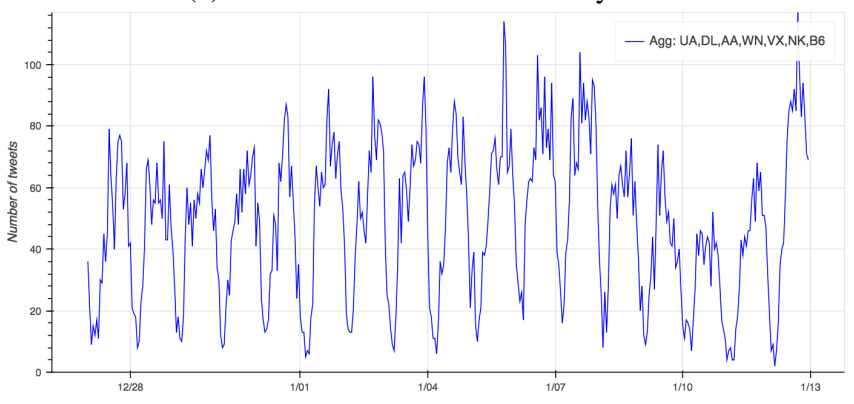

(b) Filtered with delay keywords

Fig. 14: Volume of tweets referring to airlines/airports aggregated by hour filtered by keywords

\section{Topic analysis on tweets}

A more elaborate way of exploiting information from tweets is to perform a topic analysis of the tweet database using Latent Dirichlet Allocation (LDA) [31] and comparing "normal" days (January 9th - 11th) with days where the bomb cyclone impacted the East coast (January 4th - 6th). A first step in topic analysis is to clean and format the tweets analyzed. For instance, any reference to websites or pictures is replaced by a corresponding keyword. Mentions of other users within a tweet (@ someone) and most emojis are similarly replaced. Note that "dm" means "direct message" on twitter, which is used when a user wants to take a public conversation to a private channel. As our database contains many responses from airlines, we also replaced the individual signatures of each agent by a keyword. Dates and times are also generically replaced by keywords. The resulting text is then filtered against common stop-words and words occurring only once in the whole month of January 2018 are removed. Topics are then generated using the Gensim [32] library. The best topic representation out of five runs was chosen using coherence measures introduced in [33]

Two different methods are used to study the impact of the bomb cyclone using topic analysis. In a first approach, topics are generated using the airlines-related tweets across the full month of January 2018, and then their relative importance is ranked for each set of days, see Tables IV and III. As may be expected, the topic concerning cancellations and delays went from 4th place during the normal days to 1 st place during the bomb cyclone.

\begin{tabular}{|c|c|c|}
\hline Rank & Distribution & Top 10 words \\
\hline 1 & 13.9 & $\begin{array}{c}\text { mention, flight, get, one, know, } \\
\text { plane, picture, still, website, bags }\end{array}$ \\
\hline 2 & 13.0 & $\begin{array}{c}\text { signature, sorry, thanks, flight, get, } \\
\text { hear, know, mention, time, us }\end{array}$ \\
\hline 3 & 12.3 & $\begin{array}{c}\text { mention, picture, website, flight, } \\
\text { signature, help, back, thanks, flying, get }\end{array}$ \\
\hline 4 & 11.6 & $\begin{array}{c}\text { mention, flight, get, time, thanks, } \\
\text { delayed, cancelled, flying, us, flights }\end{array}$ \\
\hline
\end{tabular}

TABLE III: Top 4 monthly topics for Jan. 9-11

\begin{tabular}{|c|c|c|}
\hline Rank & Distribution & Top 10 words \\
\hline 1 & 16.1 & $\begin{array}{c}\text { mention, flight, get, time, thanks, } \\
\text { delayed, cancelled, flying, us, flights }\end{array}$ \\
\hline 2 & 16.0 & $\begin{array}{c}\text { mention, flight, get, one, know, } \\
\text { plane, picture, still, website, bags }\end{array}$ \\
\hline 3 & 10.9 & $\begin{array}{c}\text { mention, picture, website, flight, } \\
\text { signature, help, back, thanks, flying, get }\end{array}$ \\
\hline 4 & 10.9 & $\begin{array}{c}\text { signature, sorry, thanks, flight, get, } \\
\text { hear, know, mention, time, us }\end{array}$ \\
\hline
\end{tabular}

TABLE IV: Top 4 monthly topics for Jan. 4-6

A second approach provides more specific insight regarding the bomb cyclone. Topics were determined independently for each set of days using only the tweets from the corresponding days. They are then ranked by importance on each set of days, see Tables VI and V.

\begin{tabular}{|c|c|c|}
\hline Rank & Distribution & Top 10 words \\
\hline 1 & 15.7 & $\begin{array}{c}\text { mention, flight, website, picture, } \\
\text { thanks, time, great, airline, travel, flights }\end{array}$ \\
\hline 2 & 15.1 & $\begin{array}{r}\text { mention, flight, picture, website, } \\
\text { thanks, back, get, thank, bag, signature }\end{array}$ \\
\hline 3 & 13.8 & $\begin{array}{r}\text { mention, flight, get, picture, } \\
\text { one, plane, airport, check, still, help }\end{array}$ \\
\hline 4 & 10.3 & $\begin{array}{r}\text { signature, website, please_dm, sorry, } \\
\text { dm, happy, hi, hear, bag, flight }\end{array}$ \\
\hline
\end{tabular}

TABLE V: Top 4 specific topics for Jan. 9-11

\begin{tabular}{|c|c|c|}
\hline Rank & Distribution & Top 10 words \\
\hline 1 & 22.4 & $\begin{array}{c}\text { flight, mention, get, time, flights, } \\
\text { cancelled, jfk, still, time, delayed }\end{array}$ \\
\hline 2 & 12.7 & $\begin{array}{c}\text { signature, sorry, mention, thanks, } \\
\text { us, know, please, flight, team, airport }\end{array}$ \\
\hline 3 & 11.2 & $\begin{array}{c}\text { mention, thank, website, get, } \\
\text { signature, picture, time, see, flight, airport }\end{array}$ \\
\hline 4 & 11.2 & $\begin{array}{c}\text { mention, picture, flight, thanks, } \\
\text { website, get, us, help, one, airport }\end{array}$ \\
\hline
\end{tabular}

TABLE VI: Top 4 specific topics for Jan. 4-6

Highlighting the large impact of the bomb cyclone, the keyword "jfk" suddenly appears during the corresponding time period and is associated with "cancelled" and "delayed" within the top topic. Interestingly, the tweets considered are the tweets related to airlines' handles and not to airports' handles. For the set of normal days, the topics are less specific even though they illustrate usual tweets about vacations, trips, waiting for luggage at airports as well as discussions between passengers and airlines customer services.

\section{CONClusion}

To the best of the authors' knowledge, this paper constitutes one of the first big data applications of mobile phone data 
and social media data to the analysis of the impact of large disruptions in air transportation. Leveraging two weeks of mobile location data in the United States, with more than 5 billion records per day, as well as two weeks of Twitter data, we show that mobile phones and social media can act as sensors for air traffic passengers, yielding a more complete and richer picture of the situation than traditional flight-centric measurements from the Bureau of Transportation Statistics. Thanks to these independent sources of measurements, various aviation stakeholders, who currently only have access to a partial and private view of passenger behavior, could now reliably measure system-wide passenger-centric metrics. These methods were implemented in this paper in order to provide insights on how the passenger experience was impacted at airports in the North East of the United States during the Bomb Cyclone in January 2018.

Future work will focus on implementing more automated and closer to real-time methods for analyzing and visualizing passengers experience in order to provide faster insights on the impact of different major air-traffic disturbances. The authors are also looking to scale this analysis to other countries.

\section{ACKNOWLEDGMENTS}

The authors would like to sincerely thank the Verizon Big Data and AI team for their contribution as well as Nikunj Oza from NASA-Ames for his support.

\section{REFERENCES}

[1] B. of Transportation Statistics, "Bureau of transportation statistics, about bts." [Online]. Available: http://www.rita.dot.gov/bts/about

[2] SITA, "The passenger IT trends survey," www.sita.aero/system/files/ Passenger-IT-Trends-Survey-2014.pdf, 2014.

[3] H. Nikoue, A. Marzuoli, J.-P. Clarke, E. Feron, and J. Peters, "Passenger flow predictions at sydney international airport: a data-driven queuing approach," arXiv preprint arXiv:1508.04839, 2015.

[4] NextGen Integration and Implementation Office, "Nextgen implementation plan," in Federal Aviation Administration, 2009.

[5] P. Ky and B. Miaillier, "Sesar: towards the new generation of air traffic management systems in europe," Journal of Air Traffic Control, vol. 48, no. 1, pp. 11-14, 2006.

[6] World Economic Forum, "Connected World : Transforming Travel, Transportation and Supply Chains," http://www3.weforum.org/docs, 2013.

[7] —-, "Smart travel: Unlocking economic growth and development through travel facilitation," http://www3.weforum.org/docs/GAC/2014, 2014.

[8] A. Cook, G. Tanner, S. Cristóbal, and M. Zanin, "Passenger-oriented enhanced metrics," 2012.

[9] S. Bratu and C. Barnhart, "Flight operations recovery: New approaches considering passenger recovery," Journal of Scheduling, vol. 9, no. 3, pp. 279-298, 2006.

[10] D. Wang, "Methods for analysis of passenger trip performance in a complex networked transportation system," Ph.D. dissertation, George Mason University, 2007.

[11] D. B. Work and A. M. Bayen, "Impacts of the mobile internet on transportation cyberphysical systems: traffic monitoring using smartphones," in National Workshop for Research on High-Confidence Transportation Cyber-Physical Systems: Automotive, Aviation, \& Rail, 2008, pp. 18-20.

[12] M. C. Gonzalez, C. A. Hidalgo, and A.-L. Barabasi, "Understanding individual human mobility patterns," arXiv preprint arXiv:0806.1256, 2008.

[13] V. D. Blondel, A. Decuyper, and G. Krings, "A survey of results on mobile phone datasets analysis," EPJ Data Science, vol. 4, no. 1, p. 10, 2015.
[14] V. D. Blondel, M. Esch, C. Chan, F. Clérot, P. Deville, E. Huens, F. Morlot, Z. Smoreda, and C. Ziemlicki, "Data for development: the $\mathrm{d} 4 \mathrm{~d}$ challenge on mobile phone data," arXiv preprint arXiv:1210.0137, 2012.

[15] Y.-A. de Montjoye, Z. Smoreda, R. Trinquart, C. Ziemlicki, and V. D. Blondel, "D4d-senegal: the second mobile phone data for development challenge," arXiv preprint arXiv:1407.4885, 2014.

[16] Y.-A. de Montjoye, L. Rocher, and A. S. Pentland, "bandicoot: a python toolbox for mobile phone metadata," Journal of Machine Learning Research, vol. 17, no. 175, pp. 1-5, 2016.

[17] R. W. Douglass, D. A. Meyer, M. Ram, D. Rideout, and D. Song, "High resolution population estimates from telecommunications data," EPJ Data Science, vol. 4, no. 1, p. 4, 2015.

[18] L. Alexander, S. Jiang, M. Murga, and M. C. González, "Origindestination trips by purpose and time of day inferred from mobile phone data," Transportation research part c: emerging technologies, vol. 58, pp. 240-250, 2015.

[19] J. L. Toole, S. Colak, B. Sturt, L. P. Alexander, A. Evsukoff, and M. C. González, "The path most traveled: Travel demand estimation using big data resources," Transportation Research Part C: Emerging Technologies, vol. 58, pp. 162-177, 2015.

[20] Statista, "Monthly active twitter users in the united states," https://www.statista.com/statistics/274564/ monthly-active-twitter-users-in-the-united-states/.

[21] A. Java, X. Song, T. Finin, and B. Tseng, "Why we twitter: understanding microblogging usage and communities, twitter," in Proceedings of the 9th WebKDD and 1st SNA-KDD 2007 workshop on Web mining and social network analysis. ACM, 2007, pp. 56-65.

[22] B. Krishnamurthy, P. Gill, and M. Arlitt, "A few chirps about twitter," in Proceedings of the first workshop on Online social networks. ACM, 2008, pp. 19-24.

[23] B. A. Huberman, D. M. Romero, and F. Wu, "Social networks that matter: Twitter under the microscope," arXiv preprint arXiv:0812.1045, 2008.

[24] L. Palen, K. Starbird, S. Vieweg, and A. Hughes, "Twitter-based information distribution during the 2009 red river valley flood threat, twitter," Bulletin of the American Society for Information Science and Technology, vol. 36, no. 5, pp. 13-17, 2010.

[25] S. Vieweg, A. L. Hughes, K. Starbird, and L. Palen, "Microblogging during two natural hazards events: What twitter may contribute to situational awareness," in Proceedings of the SIGCHI Conference on Human Factors in Computing Systems, ser. CHI '10. New York, NY, USA: ACM, 2010, pp. 1079-1088. [Online]. Available: http://doi.acm.org/10.1145/1753326.1753486

[26] K. Kireyev, L. Palen, and K. Anderson, "Applications of topics models to analysis of disaster-related twitter data," in NIPS Workshop on Applications for Topic Models: Text and Beyond, vol. 1. Canada: Whistler, 2009.

[27] E. F. A. S. Aude Marzuoli, Emmanuel Boidot, "Implementing and validating air passenger-centric metrics using mobile phone data," Journal of Aerospace Information Systems, 2018.

[28] Business Insider, "Jfk, laguardia airports closed as 'bomb cyclone' shuts down thousands of flights and strands travelers," http://https://www.businessinsider.com/ new-york-airports-cancel-flights-bomb-cyclone-winter-storm-2018-1.

[29] LaGuardia Airport, https://twitter.com/LGAairport/status/ 948747505763840001 .

[30] Twitter, "Twitter developper api," http://dev.twitter.com.

[31] D. M. Blei, A. Y. Ng, and M. I. Jordan, "Latent dirichlet allocation," Journal of machine Learning research, vol. 3, no. Jan, pp. 993-1022, 2003.

[32] R. Řehůřek and P. Sojka, "Software Framework for Topic Modelling with Large Corpora," in Proceedings of the LREC 2010 Workshop on New Challenges for NLP Frameworks. Valletta, Malta: ELRA, May 2010, pp. 45-50, http://is.muni.cz/publication/884893/en.

[33] M. Röder, A. Both, and A. Hinneburg, "Exploring the space of topic coherence measures," in Proceedings of the eighth ACM international conference on Web search and data mining. ACM, 2015, pp. 399-408. 